\title{
Standardised Radon Index (SRI): a normalisation of radon data-sets in terms of standard normal variables
}

\author{
R. G. M. Crockett and C. P. Holt \\ Data Analysis Group, School of Science and Technology, University of Northampton, Northampton, UK
}

Received: 22 December 2010 - Revised: 12 May 2011 - Accepted: 19 May 2011 - Published: 5 July 2011

\begin{abstract}
During the second half of 2002, from late June to mid December, the University of Northampton Radon Research Group operated two continuous hourly-sampling radon detectors $2.25 \mathrm{~km}$ apart in the English East Midlands. This period included the Dudley earthquake $\left(M_{\mathrm{L}}=5\right.$, 22 September 2002) and also a smaller earthquake in the English Channel $\left(M_{\mathrm{L}}=3\right.$, 26 August 2002). Rolling/sliding windowed cross-correlation of the paired radon time-series revealed periods of simultaneous similar radon anomalies which occurred at the time of these earthquakes but at no other times during the overall radon monitoring period. Standardising the radon data in terms of probability of magnitude, analogous to the Standardised Precipitation Indices (SPIs) used in drought modelling, which effectively equalises different non-linear responses, reveals that the dissimilar relative magnitudes of the anomalies are in fact closely equiprobabilistic. Such methods could help in identifying anomalous signals in radon - and other - time-series and in evaluating their statistical significance in terms of earthquake precursory behaviour.
\end{abstract}

\section{Introduction}

There is an increasing body of evidence which indicates that radon emissions from rocks, soils, and groundwater can provide a diagnostic tool for some geophysical processes, e.g. tidal deformations and earthquakes. In this context, it is often informative to compare two radon data-sets, e.g. variations or anomalies in radon concentrations at different locations which might be responses to common stimuli. However, this can be complicated, e.g. by the use of different

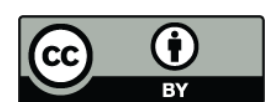

Correspondence to: R. G. M. Crockett (robin.crockett@northampton.ac.uk) detectors with different characteristics, radon concentrations being orders of magnitude different or different non-linear responses of radon emissions to stimuli due to different rock and soil properties.

As a means of investigating similarity of response to stimulus, a Standardised Radon Index (SRI) is proposed, adapted from Standardised Precipitation Index (SPI) methodologies under development at the University of Northampton (Crockett and Holt, 2010a, b). This has been piloted on a data-set known to contain simultaneous radon anomalies temporally associated with UK earthquakes (Crockett et al., 2006a, b; Crockett and Gillmore, 2010).

Changes in rock, soil, and groundwater parameters, which can influence radon emissions, are associated with the build-up and release of stresses associated with earthquakes (Meyer, 1977; Asada, 1982; Igarashi et al., 1995; Koch and Heinicke, 1994; Bolt, 2004; Walia et al., 2005, 2006). Many attempts have been made to use such variations as earthquake predictors (Finkelstein et al., 1998; Zmazek et al., 2000; Planinic et al., 2000; Bella and Plastino, 1999; Plastino et al., 2002). However, these have so far proved to be unreliable (Wakita, 1996; Kerr, 2009). Also, whilst their finding might be attributable to local conditions and an exception to a general (if variable) case, it is important to observe that Climent et al. (1999) found no relationship between radon levels and earthquakes in Japan.

In addition to well-catalogued problems associated with interpreting the influences of factors (e.g. geological, meteorological) on radon emission (e.g. Wakita, 1996; Climent et al., 1999; Chyi et al., 2001), much of this work has been impeded on two grounds. First, monitoring at a single location precludes investigation of the spatial nature of any variation. Second, the use of integrating (e.g. track-etch) detectors significantly limits observation of short-term variations (e.g. hours to days). However, whilst monitoring at two or more locations enables a determination of the degree

Published by Copernicus Publications on behalf of the European Geosciences Union. 
of localisation of radon variations, it introduces two broad sets of factors which require investigation. First, different recording equipment can have different characteristics, e.g. response times to changes in radon concentration (Crockett and Gillmore, 2010). Second, different soil properties and conditions at the monitoring sites can result in different nonlinear radon responses to (common) stimuli, such as earthquakes, and this is considered herein.

\section{Radon monitoring and results}

The solid geology in and around Northampton, in the English East Midlands, mainly consists of Lower and Middle Jurassic to Upper Lias sediments, predominantly Northamptonshire ironstone (Hains and Horton, 1969; Poole et al., 1968). There is also significant overlying unconsolidated surficial material in parts of the area (Hains and Horton, 1969; Boulton, 1992; Smith et al., 2000; Toghill, 2002). The raised radon levels in parts of the region are associated with the underlying ironstone, including the area containing the deployed radon detectors, and the variability of the overlying surficial deposits influences radon levels due chiefly to variations in gas permeability.

During the second half of 2002, the University of Northampton Radon Research Group operated two continuous hourly-sampling radon detectors $2.25 \mathrm{~km}$ apart in Northampton (Phillips et al., 2004). This period included the Dudley earthquake of 22 September 2002 (23 September 2002 BST), which was widely noticed by members of the public in the Northampton area (Crockett et al., 2006a; Gillmore and Crockett, 2008). This period also included an English Channel earthquake, 26 August 2002, and the Manchester earthquake swarm, 21-29 October 2002, neither of which were noticed in the Northampton area. The earthquake incidence data are summarised in Table 1 (BGS (http://www.bgs.ac.uk), 2003; ANSS (http://quake. geo.berkeley.edu/anss/), 2008).

During this monitoring period, two hourly-sampled timeseries of radon readings were obtained, one from each deployed detector (both Durridge RAD7s). These paired timeseries have been analysed for evidence of simultaneous similar anomalies, anomalies in the time-domain in addition to or instead of anomalies in magnitude. The working hypothesis for this is that a big disturbance, such as an earthquake, occurring at a relatively large distance ( $\sim 100 \mathrm{~km}$ or more $)$ compared to the detector separation $(2.25 \mathrm{~km})$ could be expected to produce simultaneous similar radon anomalies (Crockett et al., 2006a). Conversely, any anomalies which are neither simultaneous nor similar are more likely to arise from stimuli local to individual monitoring locations. Under circumstances where the detector separation is greater in comparison to the stimulus, the assumption of simultaneity would have to be modified to account for different signal pathlengths.
Table 1. Earthquake Data, July-October 2002, (BGS, ANSS).

\begin{tabular}{llccc}
\hline $\begin{array}{l}\text { Location } \\
\text { Location }\end{array}$ & $\begin{array}{l}\text { Date/Time } \\
(\mathrm{UTC} / \mathrm{GMT})\end{array}$ & $\begin{array}{l}\text { Mag. } \\
\left(M_{\mathrm{L}}\right)\end{array}$ & $\begin{array}{c}\text { Dist } \\
(\mathrm{km})\end{array}$ & $\begin{array}{c}\text { Depth } \\
(\mathrm{km})\end{array}$ \\
\hline English Channel & 26 Aug 2002 23:41 & 3.0 & 250 & 4.0 \\
\hline $\begin{array}{l}\text { Dudley main } \\
\text { aftershock }\end{array}$ & 22 Sep 2002 23:53 & 5.0 & 91 & 9.4 \\
aftershock & 24 Sep 2002 03:32 & 3.2 & 90 & 9.3 \\
\hline Manchester & 21 Oct 2002 07:45 & 3.7 & 156 & 5.0 \\
Manchester & 21 Oct 2002 11:42 & 4.3 & 165 & 5.0 \\
Manchester & 22 Oct 2002 03:39 & 2.9 & 163 & 5.0 \\
Manchester & 22 Oct 2002 12:28 & 3.5 & 161 & 4.2 \\
Manchester & 23 Oct 2002 01:53 & 3.3 & 163 & 5.0 \\
Manchester & 24 Oct 2002 08:24 & 3.8 & 164 & 3.7 \\
Manchester & 24 Oct 2002 15:46 & 2.8 & 164 & 5.0 \\
Manchester & 25 Oct 2002 00:19 & 2.5 & 164 & 5.0 \\
Manchester & 25 Oct 2002 00:20 & 2.5 & 164 & 5.0 \\
Manchester & 25 Oct 2002 17:24 & 2.6 & 164 & 5.0 \\
Manchester & 29 Oct 2002 04:42 & 3.1 & 164 & 5.0 \\
\hline
\end{tabular}

The time-series are shown in Fig. 1 for the five-month period, July-November 2002; Fig. 1a shows TS1a, Fig. 1b shows TS1b and Fig. 1c shows both time-series normalised to unit mean to assist visual comparison in this and subsequent figures. In summary, both time-series are closely lognormally distributed, with correlation coefficients to lognormal distributions of $>0.91$, although TS1a (arithmetic mean $1259 \mathrm{~Bq} \mathrm{~m}^{-3}$, geometric mean $1125 \mathrm{~Bq} \mathrm{~m}^{-3}$ ) is typically 30 40 times greater than $\mathrm{TS} 1 \mathrm{~b}$ (arithmetic mean $46.3 \mathrm{~Bq} \mathrm{~m}^{-3}$, geometric mean 29.2 $\mathrm{Bq} \mathrm{m}^{-3}$ ) (Crockett et al., 2006a). Both time-series are characterised by noisy, non-stationary $24 \mathrm{~h}$ cycles having short autocorrelation lengths, ca. 1-2 days, as shown in Fig. 2; with out-of-phase daily maxima typically occurring at ca. 18:00 GMT and 16:00 GMT in TS1a and TS1b respectively (Crockett et al., 2006a). There is weak evidence of seven-day cycles, typical of anthropogenic influences, and also evidence of some longer-period variations, durations ca. 15 and 30 days, which are possibly attributable to lunar-tidal influences (Crockett et al., 2006a, b). With the exception of rainfall, behind which TS1a and TS1b lag by 14 and 10 days respectively, there are no observed meteorological dependencies (Crockett et al., 2006a).

\subsection{Correlation analysis}

The paired time-series were investigated for simultaneous similar features using a rolling/sliding, windowed crosscorrelation technique (Crockett et al., 2006a). In brief, for the desired window duration, the window is rolled/slid forwards throughout the entire duration of the paired time-series, one interval at a time, cross-correlating at every position. Thus, rolling/sliding a 1-day window results in correlating successive sets of 24 hourly readings, a 2-day window correlates 


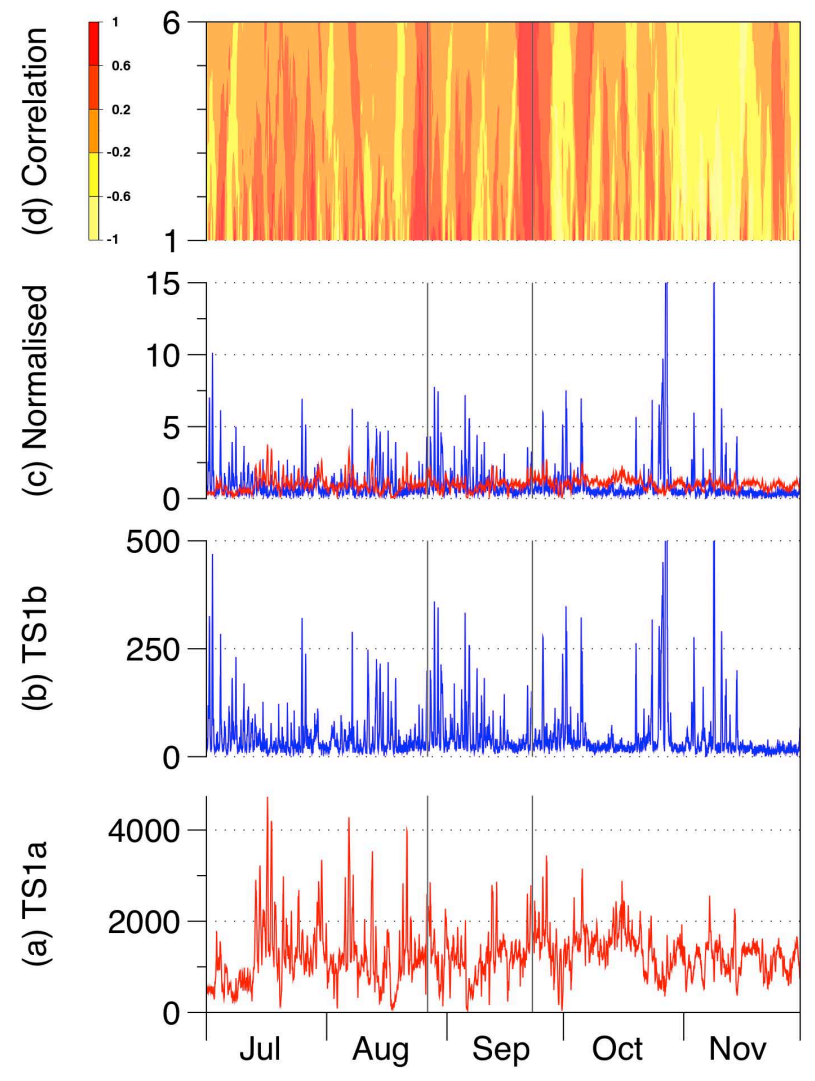

Date (month, 2002)

Fig. 1. Radon Time-Series, July-November 2002; (a) TS1a (red) hourly data, (b) TS1b (blue) hourly data, (c) TS1a (red) and TS1b (blue) normalised hourly data, (d) rolling/sliding windowed crosscorrelation 1-6 day windows. The vertical grey lines show the main earthquake timings.

sets of 48 points, etc. The result is a time-series of correlation coefficients indicating when the time-series are behaving similarly or otherwise. Originally, windows of up to 30-day duration were used but for this analysis the majority of the useful information is revealed by windows of duration up to ca. 6-day duration, as shown contour-plotted in Fig. 1d. This shows two distinct periods of high positive correlation evident around 25-27 August and 21-23 September 2002 (e.g. 3-day correlation coefficients of 0.87 for both periods, compared to the 5-month value of -0.09 ) within a scatter of shorter-periods (revealed only in shorter windows) of high positive correlation on a general slight trend from positive to negative cross-correlation. Investigation of these two distinct periods reveals two anomalous ca. 5-day sections of the overall 175-day paired time-series where the underlying, noisy, non-stationary 24 -h cycles synchronise and are more clearly defined, i.e. simultaneous similar radon anomalies of approximately 6-h duration in the paired time-series. Such sequences are not apparent elsewhere in the data.

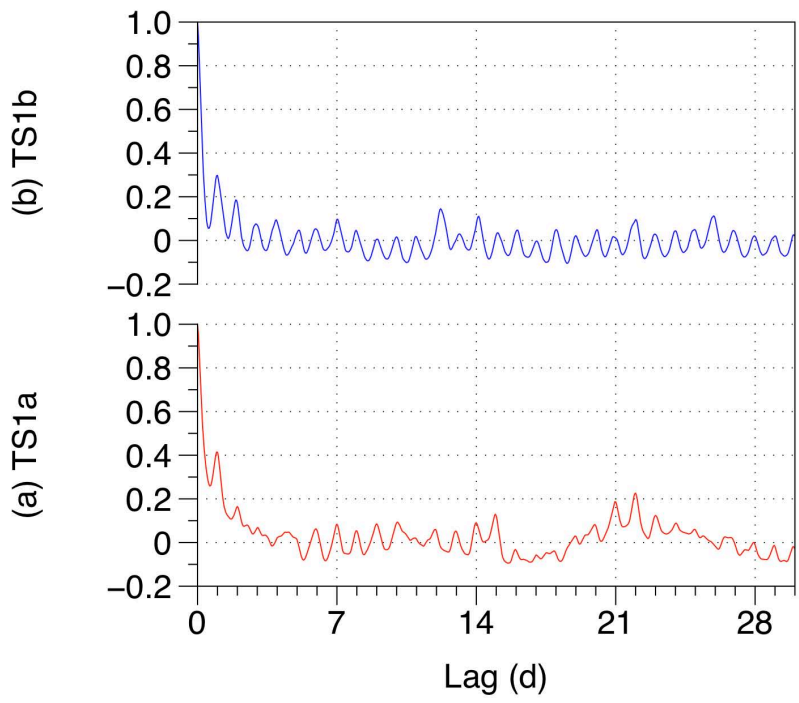

Fig. 2. Autocorrelation of the Radon Time-Series; (a) TS1a (red), (b) TS1b (blue).

These two sequences are shown in Figs. 3a and 4a respectively (normalised radon, as Fig. 1c). The first, less distinct, sequence (Fig. 3) is temporally associated with the English Channel earthquake; the second, more distinct, sequence (Fig. 4) is temporally associated with the Dudley earthquake. In both figures, the earthquake timings are indicated by vertical grey lines. Whilst the normalisation of the radon data facilitates (visual) comparison, it does not inform with regard to the potentially different non-linear radon responses at the two locations. However, it is possible to compare radon responses by considering the probability of occurrence of radon concentrations.

\subsection{Standardising radon time-series}

In general, in order to maximise a comparison of two (or more) radon time-series, as well as taking account of different equipment characteristics (Crockett and Gillmore, 2010), ideally account must also be taken of different radonemission properties of the rock/soil/water systems at the monitoring locations. In this case, both time-series were recorded using Durridge RAD7s, so there is no necessity to "filter" the data to minimise differences due to equipment responses. Indeed, not only would this not address any potential differences in radon-emission, it would potentially unnecessarily remove information arising from differences in radon-emission. The Standardised Radon Index (SRI) is proposed to address this and, in representing the data in terms of probability of occurrence, as described below, effectively standardises any difference in radon response, thereby facilitating comparison. The SRI can be considered as a rerepresentation of a radon time-series in terms of probability of magnitude and is mathematically similar to the Standardised Precipitation Index (SPI). 


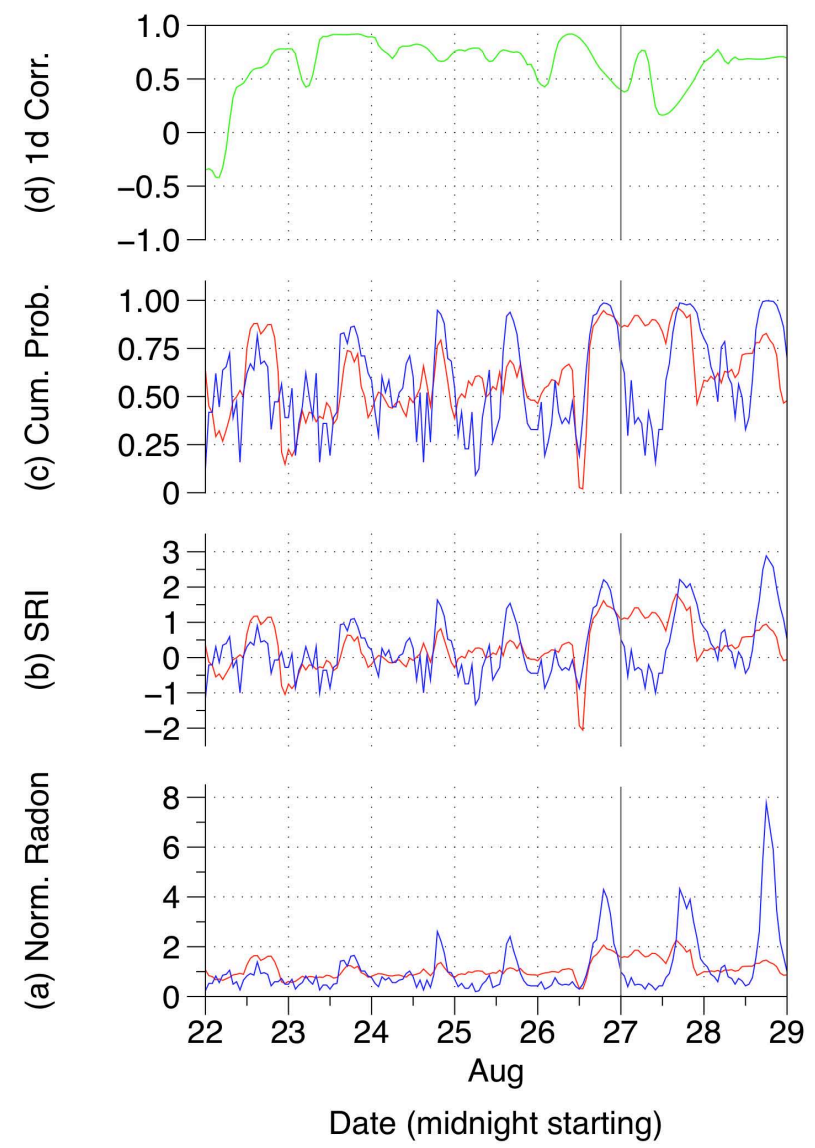

Fig. 3. Late August Radon Anomalies: (a) Normalised Radon, (b) SRI, (c) Cumulative Probability, (d) windowed 1-day correlation. TS1a and TS1b are shown in red and blue, respectively, in graphs $(\mathbf{a}-\mathbf{c})$. The vertical grey line shows the timing of the English Channel Earthquake of 26 August 2002.

\subsubsection{Standardised Precipitation Index (SPI)}

SPIs were first proposed by McKee et al. in 1993, and can be summarised as a normalisation of precipitation data in terms of standard normal random variables. In effect, variations in the data are presented in terms of abscissae of the standard normal distribution via an equiprobability mapping from the cumulative probability distribution of the data. Thus, periods of the same relative drought (or high-precipitation) in different precipitation regimes have the same SPI: the same SPI in different data-sets represents the same relative drought/highprecipitation across different precipitation regimes.

The standard formalisation of SPIs, as proposed by McKee et al. (1993), involves an equiprobability mapping between a gamma distribution, used to fit the data, and the standard normal distribution, from which SPIs are derived as abscissae. The equiprobability mapping can be eliminated where the precipitation data can be transformed to normally-distributed data from which SPIs can be calculated directly, e.g. precipitation data with (truncated) square- or cube-root normal distributions (Kendall, 1960; Dingens and Steyaert, 1971;

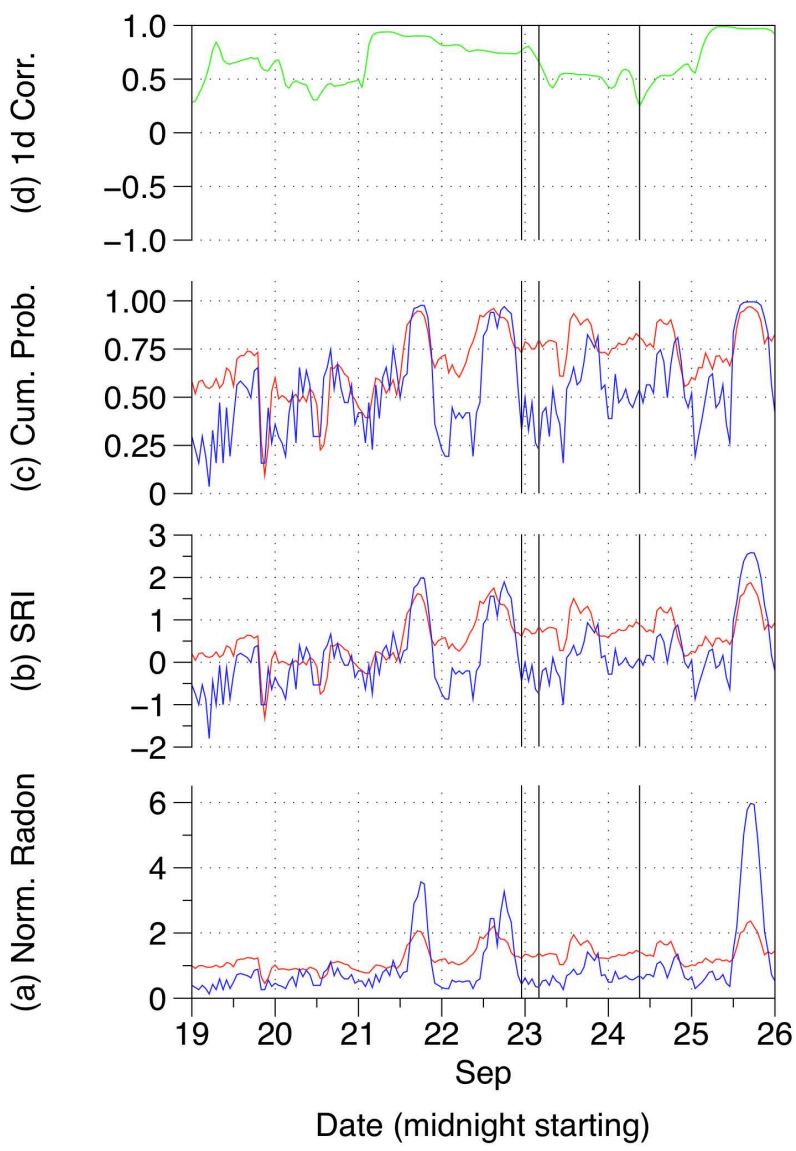

Fig. 4. Late September Radon Anomalies: (a) Normalised Radon, (b) SRI, (c) Cumulative Probability, (d) windowed 1-day correlation. TS1a and TS1b are shown in red and blue respectively in graphs $(\mathbf{a}-\mathbf{c})$. The vertical grey lines show the timing of the Dudley Earthquake of 22 September 2002 and its two aftershocks.

Crockett and Holt, 2010a; Fu et al., 2010). The underlying assumption is that the (cumulative) probability of a transformed datum within the transformed data-set is equal to the (cumulative) probability of a raw datum within the raw data-set. In drought and precipitation work, for example, for square- or cube-root normally distributed precipitation data, the SPIs can be obtained directly by mapping the normally distributed square- or cube-roots, respectively, onto the standard normal distribution. More generally, other (truncated) root-normal distributions can be used to model precipitation data, from which SPIs can be calculated directly avoiding an equiprobability mapping (Crockett and Holt, 2010a, b).

\subsubsection{Standardised Radon Index (SRI)}

Transferring the concept of SPIs to radon, where data-sets are generally lognormally distributed, is straightforward and does not require an equiprobability mapping (where the data are lognormally distributed). Simply, SRIs can be calculated directly from the logarithms of the data, taking care 
to express any zero-data as an appropriately small fraction of the detector minimum threshold before taking the logarithms. In essence, it is the standard-normal $z$-mapping, as widely used in statistics, of the logarithms of the data and the SRIs are abscissae of the standard normal distribution.

Graphs $b$ in each of Figs. 3 and 4 show the Standardised Radon Indices (SRIs), and graphs c show the corresponding cumulative probabilities. The 1-day windowed correlation coefficients are shown in graphs $\mathrm{d}$ for reference with Fig. 1. It is clear from both the SRIs and the cumulative probabilities that the simultaneous anomalies are more similar in terms of probability of occurrence than in relative amplitude.

\section{Discussion}

Although the mathematics of SPIs and SRIs are similar, the usages and contexts differ. SPIs are calculated for periods (such as months, quarters, and half years) because precipitation data are generally aggregated, but often precipitation has seasonal variations within such timescales and droughts have durations within such timescales. For SRIs, however, the data are not aggregated and each datum is converted to its SRI, effectively a standardised magnitude. The immediate use of SRIs is to compare transient responses in radonemission properties via probability of occurrence, but SPIlike use to compare period-aggregated data is also possible.

Figures 3 and 4 show two sections of paired time-series in terms of normalised radon data, SRIs, and cumulative probabilities. The normalised radon data show synchronised peaks of similar durations but dissimilar relative amplitude, the TS1b (normalised, relative) peaks typically being larger than the TS1a ones. However, when the data are presented in terms of probability of occurrence, either as SRIs (standardised magnitudes) or cumulative probabilities, the peaks in TS1a and TS1b are more similar. Assuming that both timeseries are responding to common stimuli during these two periods, it can be inferred that the radon-emission response at location-a is damped compared to the response at locationb: i.e. similar probabilities but different relative magnitudes. Also, as is clear from Fig. 4, presentation of the data in terms of SRIs or cumulative probabilities reveals a simultaneous dip ca. $48 \mathrm{~h}$ before the Dudley earthquake more clearly, as an equal-probability response, than in the normalised radon data.

Despite the greater magnitude of some of the Manchester earthquakes, and their relative proximity compared to the English Channel earthquake, no such sequences of similar anomalies have been observed at the time of the Manchester earthquakes. It is possible that there are no such radon anomalies temporally associated with these earthquakes, due to the geology at and between Manchester and Northampton, for example, or it is possible that there are anomalies but these are blurred by a combination of geological factors and the swarm nature of the earthquakes.
In previous investigations, we have applied moving averages to reduce the effects of drift/mismatch in sampling times (for same sampling period), and also techniques such as Empirical Mode Decomposition (EMD) to de-noise time-series and reduce the effects of different detector responses. However, both these techniques potentially result in a loss of information and neither accounts for different non-linear radon responses to similar or common stimuli as do the SRIs we propose.

\section{Conclusions}

In representing a radon time-series in terms of SRIs, it is possible to directly compare features and anomalies in terms of probability, thus accounting for some of the different nonlinearities of radon-emission response of different rocks and soils (and, potentially, different detectors). This enables a fuller comparison of the paired time-series during the periods of high positive cross-correlation. It does not, however, significantly effect the outcome of the rolling/sliding windowed cross-correlation because, whilst differentially extending or compressing the data magnitudes according to probability, it does not alter the basic shape, which is what correlation compares and quantifies.

For lognormally-distributed data-sets, SRIs are easy to calculate from the logarithms of the data. For non-lognormally distributed data-sets, other transformations and SPI-type equiprobability mappings afford routes of calculation.

Whilst this is work in progress, these results demonstrate that there is the potential for such techniques to enhance the possibility that simultaneous real-time monitoring of radon levels for short-term simultaneous anomalies at several locations in earthquake areas might provide the core of an earthquake prediction method.

Acknowledgements. The authors gratefully acknowledge members of the University of Northampton Radon Research Group for the collection of the data and also DEFRA (UK) for funding the research under which the 2002 data were collected (EPG 1/4/72, RW 8/1/64).

Edited by: F. Perrier

Reviewed by: J.-C. Sabroux and another anonymous referee

\section{References}

Asada, T.: Earthquake Prediction Techniques: Their Application in Japan, University of Tokyo Press, Japan, 1982.

Bella, F. and Plastino, W.: Radon time series analysis at LNGS, II, LNGS Annual Report 1999, Gran Sasso National Laboratory, Italy, 199-203, 1999.

Bolt, B. A.: Earthquakes, 5th Edn., W.H. Freeman \& Co, New York, USA, 2004.

Boulton, G. S.: Quaternary, In: Geology of England and Wales, edited by: Duff, P. McL, D., Smith, A. J. (Eds.), The Geological Society, London, 413-444, 1992. 
Chyi, L., Chou, C.-Y., Yang, F., and Chen, C.-H.: Continuous Radon Measurements in Faults and Earthquake Precursor Pattern Recognition, Western Pacific Earth Sciences, 1(2), 227-246, 2001.

Climent, H., Tokonami, S., and Furukawa, M.: Statistical Analysis Applied to Radon and Natural Events, Proc. Radon in the Living Environment, Athens, Greece, April 1999, 241-253, 1999.

Crockett, R. G. M. and Gillmore, G. K.: Spectral-decomposition techniques for the identification of radon anomalies temporally associated with earthquakes occurring in the UK in 2002 and 2008, Nat. Hazards Earth Syst. Sci., 10, 1079-1084, doi:10.5194/nhess-10-1079-2010, 2010.

Crockett, R. G. M. and Holt, C. P.: Direct data-transformation calculation of Standardised Precipitation Indices, Geophys. Res. Abstr., Vol. 12, EGU2010-1959, EGU General Assembly, Vienna, May, 2010a.

Crockett, R. G. M. and Holt, C. P.: Standardised Radon Index: a normalisation of radon data-sets in terms of standard normal variables, Geophys. Res. Abstr., 12, EGU2010-1839, EGU General Assembly, Vienna, May, 2010b.

Crockett, R. G. M., Gillmore, G. K., Phillips, P. S., Denman, A. R., and Groves-Kirkby, C. J.: Radon Anomalies Preceding Earthquakes Which Occurred in the UK, in Summer and Autumn 2002, Sci. Total Environ., 364, 138-148, 2006 a.

Crockett, R. G. M., Gillmore, G. K., Phillips, P. S., Denman, A. R., and Groves-Kirkby, C. J.: Tidal Synchronicity of Built Environment radon levels in the UK, Geophys. Res. Lett., 33, L0538, doi:10:2005GL024950, 2006b.

Dingens, P. and Steyaert, H.: A Truncated Square-Root-Normal Distribution Applied To Monthly Precipitation Totals For Ghent (Belgium), Bulletin of the International Association of Scientific Hydrology, XVI, 2(6), 79-85, 1971.

Finkelstein, M., Brenner, S., Eppelbaum, L., and Ne'eman, E.: Identification of Anomalous Radon Concentrations Due to Geodynamics Processes by Elimination of Rn Variations Caused by Other Factors, Geophys. J. Int., 133, 407-412, 1998.

Fu, G., Viney, N. R., and Charles, S. P.: Evaluation of various root transformations of daily precipitation amounts fitted with a normal distribution for Australia. Theor. Appl. Climatol., 99, 229238, doi:10.1007/s00704-009-0137-6, 2010.

Gillmore, G. K. and Crockett, R. G. M.: Radon anomalies associated with UK earthquakes which occurred in the summer and autumn of 2002. Geophys. Res. Abstr., Vol. 10, EGU2008A-03991. SREF-ID: 1607-7962/gra/EGU2008-A-03991, EGU General Assembly, Vienna, April, 2008.

Hains, B. A. and Horton, A.: British Regional Geology Central England, 3rd Edn., HMSO, 1969

Igarashi, G., Saeki, S., Takahata, N., Sumikawa, K., Tasaka, S., Sasaki, Y., Takahashi, M., and Sano, Y.: Ground-Water Radon Anomaly Before the Kobe Earthquake in Japan, Science, 269, 60-61, doi:10.1126/science.269.5220.60, 1995.
Kendall, G. R.: The cube-root normal distribution applied to Canadian monthly rainfall totals, Association Int'l d'Hydrologie Scientifique, Publ'n no. 53, 250-260, 1960.

Kerr, R. A.: After the Quake, in Search of the Science - or even good prediction, Science, 324(5925), p. 322, doi:10.1126/science.324.5925.322, 2009.

Koch, U. and Heinicke, J.: Radon Behaviour in Mineral Spring Water of Bad Bramburgh (Vogtland, Germany) in the Temporal Vicinity of the 1992 Rörmond Earthquake, the Netherlands, Geologie en Mijnbouw, 73, 399-406, 1994.

McKee, T. B., Doesken, N. J., and Kleist, J.: The relationship of drought frequency and duration to time scales. Preprints, 8th Conference on Applied Climatology, 179-184, 17-22 January, Anaheim, California, 1993.

Meyer, L. L.: California Quake, Sherbourne Press, Nashville, USA, 1977.

Planinic, J., Radolic, V., and Culo, D.: Searching for an Earthquake Precursor: temporal variations of radon in soil and water, Fizika B, 9(2), 75-82, 2000.

Plastino, W., Bella, F., Catalano, P., and Di Giovambattista, R.: Radon groundwater anomalies related to the Umbria-Marche, September 26, 1997, Earthquakes, Geofis. Int., 41(4), 369-375, 2002.

Poole, E. G., Williams, B. J., and Hains, B. A.: Geology of the Country around Market Harborough. Institute of Geological Sciences Memoirs of the Geological Survey of Great Britain, England and Wales, HMSO, 1968.

Smith, K. A., Gillmore, G. K., and Sinclair, J. M.: Sediments and Ostracoda from Courteenhall, Northamptonshire, U.K. and their implications for the depositional environment of the Pleistocene Milton Formation, P. Geologist Assoc. 111, 253-263, 2000.

Toghill, P.: The Geology of Britain: an Introduction, Airlife, Wiltshire, UK, 2002.

Phillips, P. S., Denman, A. R., Crockett, R. G. M., Groves-Kirkby, C. J., and Gillmore, G. K.: Comparative analysis of weekly vs. three-monthly radon measurements in dwellings, DEFRA commissioned research for radioactive substances division, Report DEFRA/RAS/03.006., 2004.

Wakita, H.: Geochemical Challenge to Earthquake Prediction, Proc. Natl. Acad. Sci. USA, 93, 3781-3786, 1996.

Walia, V., Virk, H. S., Yang, T. F., Mahajan, S., Walia, M., and Bajwa, B. S.: Earthquake prediction studies using radon as a precursor in N-W Himalayas, India: A case study, Terrestrial, Atmos. Ocean. Sci., 16(4), 775-804, 2005.

Walia, V., Virk, H. S., and Bajwa, B. S.: Radon precursory signals for some earthquakes of magnitude $>5$ occurred in $\mathrm{N}-\mathrm{W}$ Himalaya, Pure Appl. Geophys., 163(4), 711-721, 2006.

Zmazek, B., Vaupotic, J., Zivcic, M., Premru, U., and Kobal, I.: Radon Measurements for Earthquake Prediction in Slovenia, Fizika B, 9(3), 111-118, 2000. 